This document is the accepted manuscript version of the following article:

Merg, A. D., Touponse, G., van Genderen, E., Zuo, X., Bazrafshan, A., B1um, T., ... Contice11o, V. P. (2019). 2D crystal engineering of nanosheets assembled from helical peptide building blocks. Angewandte Chemie International Edition, 58(38), 13507-13512. https://doi.org/10.1002/anie.201906214

WILEY-VCH

\title{
2D Crystal Engineering of Nanosheets Assembled from Helical Peptide Building Blocks
}

\author{
Andrea D. Merg, ${ }^{[a]}$ Gavin Touponse,${ }^{[a]}$ Eric van Genderen, ${ }^{[b]}$ Xiaobing Zuo, ${ }^{[c]}$ Alisina Bazrafshan, ${ }^{[a]}$ \\ Thorsten Blum, ${ }^{[b]}$ Spencer Hughes, ${ }^{[a]}$ Khalid Salaita, ${ }^{[a]}$ Jan Pieter Abrahams, ${ }^{[b, d, e]}$ and Vincent $P$. \\ Conticello*[a]
}

\begin{abstract}
The successful integration of $2 \mathrm{D}$ nanomaterials into functional devices hinges on developing fabrication methods that afford hierarchical control across length-scales of the entire assembly. Here, we demonstrate structural control over a class of crystalline $2 \mathrm{D}$ nanosheets assembled from collagen triple helices. By lengthening the triple helix unit via sequential additions of Pro-HypGly triads, we impart sub-angstrom tuning over the 2D lattice. These subtle changes influence the overall nanosheet size, which can be adjusted across the mesoscale size regime. The internal structure is observed via cryo-TEM with direct electron detection, which provides real-space high-resolution images, in which individual triple helices comprising the lattice can be clearly discerned. These results establish a general strategy for tuning the structural hierarchy of $2 \mathrm{D}$ nanomaterials that employ rigid, cylindrical structural units.
\end{abstract}

Owing to their ultrathin and planar geometry, two-dimensional (2D) nanoscale materials possess intrinsic physical properties that make them suitable for a diverse range of applications. ${ }^{[1]}$ Catalyzed by the discovery of graphene and its unique properties, ${ }^{[2]}$ numerous 2D nanomaterials of various compositions have been synthesized. However, systematic control of their structural hierarchy across a range of lengthscales remains a significant challenge. This is especially evident in the context of bottom-up assembly methods for free-standing 2D nanostructures. Typical yields comprise polymorphic assemblies, with little control over their lateral dimensions, which limits further materials processing. Harvesting materials of predetermined uniform sizes would streamline their incorporation into functional devices. Consequently, new assembly methods, which afford hierarchical control over the entire assembled product (e.g., from the nano- to mesoscale) are critical for realizing their potential applications.

Recent 2D fabrication methods have employed sequenceprogrammable oligomers such as block copolymers, ${ }^{[3]}$ peptides, ${ }^{[4]}$ peptoids, ${ }^{[5]}$ and nucleic acids. ${ }^{[6]}$ Of these, peptides represent a richly studied supramolecular building block. They

\footnotetext{
[a] Dr. A. D. Merg, G. Touponse, A. Bazrafshan, S. Hughes, Prof. K. Salaita, Prof. V. P. Conticello

Department of Chemistry, Emory University

1515 Dickey Drive, Atlanta, GA 30322 (USA)

E-mail:vcontic@emory.edu

[b] Dr. E. van Genderen, Dr. T. Blum, Prof. J. P. Abrahams

Paul Scherrer Institut

$\mathrm{CH}-5232$ Villigen, $\mathrm{PSI}$ (Switzerland)

[c] Dr. X. Zuo,

$X$-ray Science Division, Argonne National Laboratory

9700 South Cass Avenue, Lemont, IL 60439 (USA)

[d] Center for Cellular Imaging and NanoAnalytics, Biozentrum, University of Basel

$\mathrm{CH}-4058$, Basel, Switzerland

[e] Leiden Institute of Biology

Sylviusweg 72, 2333 BE Leiden, The Netherlands
}

Supporting information for this article is given via a link at the end of the document. possess excellent chemical diversity, and predictable assembly behavior, which can be encoded through rational alterations to the primary structure. ${ }^{[7]}$ However, the intrinsic chirality of amino acids and proclivity to unidirectional growth via $\mathrm{H}$-bonding interactions precludes propagation in two dimensions. To circumvent this predisposition, researchers have utilized helical structural motifs (e.g. coiled coils, collagen triple helices, and alpha helices) to bias self-assembly in the lateral dimensions (Figure 1a). ${ }^{[4-j]}$ These structural units can be considered as 'rigid rod' tectons Programming interactions between the faces of these rod-like units can direct their lateral assembly.

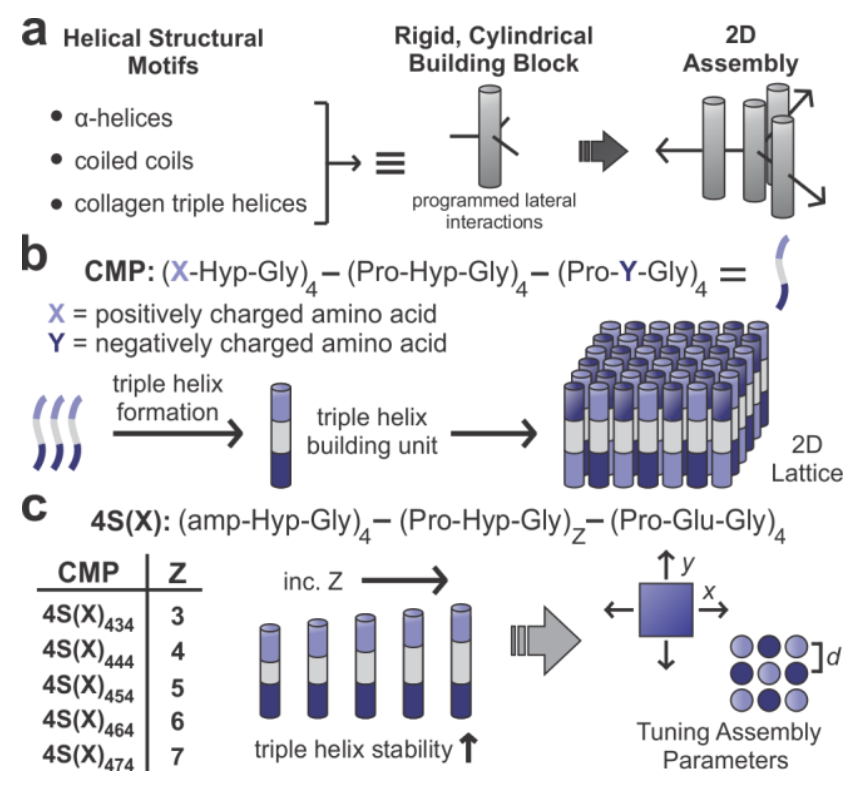

Figure 1. (a) 2D assemblies constructed from rigid, helical peptide building blocks. (b) Amino acid sequence of CMPs comprise positively charged, neutral, and negatively charged triads. Their folding into collagen triple helices gives rise to the triple helix building unit. Antiparallel packing of triple helices into a tetragonal 2D lattice is facilitated via alignment of complementary charge interactions between adjacent triple helices. (c) $\mathbf{4} \mathbf{S}(\mathbf{X})$ peptide series with varying number, $\mathrm{Z}$, of Pro-Hyp-Gly triads incorporated into the central block (amp = 4S-aminoproline). We hypothesize that incremental changes to CMP length may offer hierarchical control over the assembly parameters.

Exploiting this design strategy, we have reported the construction of crystalline nanosheets assembled from collagenmimetic peptides (CMPs). ${ }^{[4 h, 4 i]}$ These peptides comprise three sequential blocks having positively charged, neutral, and negatively charged triads. The folding of these peptides into collagen triple helices facilitates their packing into a $2 \mathrm{D}$ lattice via Coulombic interactions between oppositely charged residues on adjacent triple helices (Figure $1 \mathrm{~b}$ ). The pristine and simple nanosheet construction leverages these materials as an ideal 

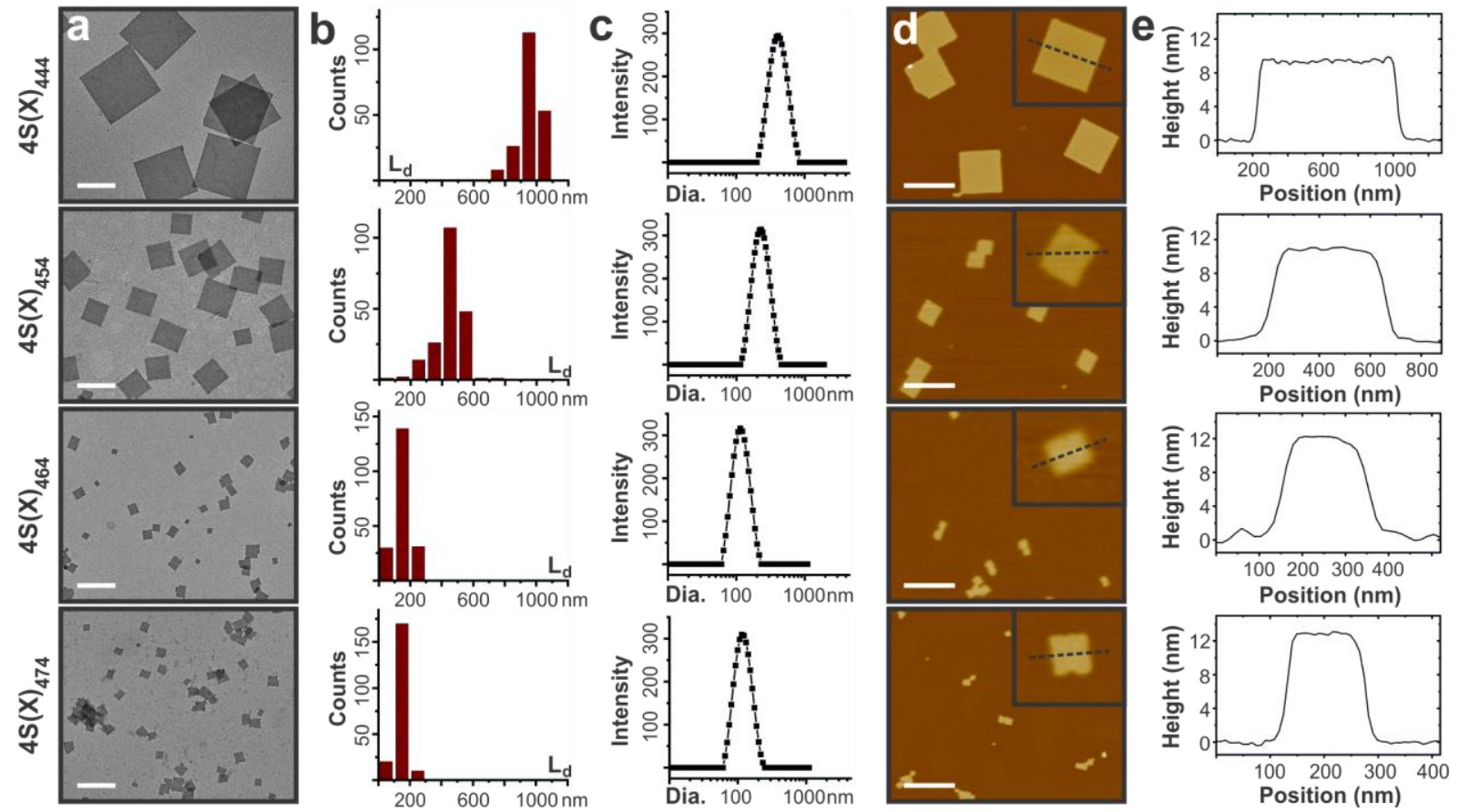

Figure 2. Characterization of $\mathbf{4 S}(X)_{444}, \mathbf{4 S}(X)_{454}, \mathbf{4 S}(X)_{464}$, and $\mathbf{4 S}(X)_{474}$ nanosheets: (a) TEM images (scale bar $\left.=500 \mathrm{~nm}\right)$; (b) $L_{d}$ distribution of $C M P$ nanosheets based on TEM (200 counts each); (c) DLS spectra; (d) AFM images (scale bar = $1 \mu \mathrm{m}$ ); and (e) height trace of nanosheet shown in the inset of (d).

platform for studying how tuning the structural properties of helical building blocks influences the internal packing (i.e. nanoscale order) and overall assembly architecture (i.e mesoscale order) for this material class. Understanding these factors will afford broad engineering principles for designing $2 \mathrm{D}$ assemblies that are constructed from rigid (super)-helical structural motifs and provides a straightforward route for tailoring their structural properties.

Here, we present a family of CMPs with a varying number of canonical pro-triple helix forming Pro-Hyp-Gly triads comprising the central block (Figure 1c). We hypothesized that this series would provide a systematic level of understanding of the factor(s) that dictate structural changes of these 2D assembly systems involving rod-like protomers. Increasing the peptide length would influence a variety of factors, in particular triple helix stability and thickness of the resulting nanosheets, however, it was unclear as to whether it would influence lateral size and sheet dispersity.

CMPs were synthesized and purified using established protocols and their compositions were confirmed via mass spectrometry (see SI for details, Figures $\mathrm{S} 1,2$ ). Initial assembly experiments involved dissolving CMPs $(4 \mathrm{mg} / \mathrm{mL})$ in $20 \mathrm{mM} 3-$ (N-morpholino)propanesulfonic acid buffer (MOPS, pH 7.0), heating to $90^{\circ} \mathrm{C}$, and cooling to $4^{\circ} \mathrm{C}$ or room temperature. The assemblies were allowed to assemble for 2 weeks.

Confirming our previous report, $\mathbf{4 S}(\mathbf{X})_{\mathbf{4 4 4}}$ (previously termed NSIII) assembles into square nanosheets of homogenous size at $4^{\circ} \mathrm{C}$ (diagonal length, $L_{d}=953 \pm 72 \mathrm{~nm}$; Figures 2a,b).$^{[4]}$ $\mathbf{4 S}(X)_{454}$ assembles into smaller nanosheets $\left(L_{d}=443 \pm 90 \mathrm{~nm}\right)$ at RT (Figures 2a,b). Their formation at room temperature is credited to the extra Pro-Hyp-Gly triad. $\mathbf{4 S}(X)_{464}$ and $\mathbf{4 S}(X)_{474}$ also assemble into nanosheets at room temperature, but the sheets appear to coalesce forming larger "raft-like" assemblies (Figure S3). This could be due to the greater contour length associated with longer Pro-Hyp-Gly blocks, which may promote lateral interaction between the incipient nanosheets. To prevent raft formation, we repeated the assembly experiments at lower [CMP]. $\mathbf{4 S}(X)_{464}$ and $\mathbf{4 S}(\mathbf{X})_{474}$, assembled at $1 \mathrm{mg} / \mathrm{mL}$, form discrete nanosheets of similar dimensions $\left(L_{d}=153 \pm 44 \mathrm{~nm}\right.$ and $149 \pm 36 \mathrm{~nm}$, respectively; Figure 2a,b). Finally, few nanosheets were observed for $\mathbf{4 S}(\mathbf{X})_{434}$ at $4^{\circ} \mathrm{C}$ indicating that there is a threshold peptide length necessary for assembly to occur at this [CMP] (Figure S4). Doubling the concentration (8 $\mathrm{mg} / \mathrm{mL}$ ) yields polymorphic nanosheets in all dimensions, likely due to the high [CMP], and therefore we did not fully characterize their assembly (Figure S5). These results suggest that peptide concentration and assembly temperature have a significant influence on the optimal conditions for nanosheet formation, which may be interpreted in terms of these effects on triple helix stability.

The differences in sheet sizes observed via TEM was confirmed in situ using dynamic light scattering (DLS). Although, the Stokes-Einstein relationship is not valid for $2 \mathrm{D}$ assemblies, the population of nanosheets could still be fit to a single curve that is consistent with a uniform size distribution of selfassembled species (Figure 2c). DLS data confirm the decrease in sheet size with increasing Pro-Hyp-Gly repeats within the core block.

Atomic force microscopy (AFM) analysis of the nanosheets reveal sheet thicknesses of $9.4 \pm 0.1 \mathrm{~nm}, 11.0 \pm 0.3 \mathrm{~nm}, 11.9 \pm$ $0.5 \mathrm{~nm}$, and $12.8 \pm 0.2 \mathrm{~nm}$ for $\mathbf{4 S}(\mathbf{X})_{444}, \mathbf{4 S}(\mathbf{X})_{454}, \mathbf{4 S}(\mathbf{X})_{464}$, and $\mathbf{4 S}(\mathbf{X})_{474}$ sheets, respectively (Figures $2 \mathrm{~d}, \mathrm{e}$ and $\mathrm{S6}, 7$ ). These 
measurements agree with theoretical thicknesses of $10.3 \mathrm{~nm}$, $11.2 \mathrm{~nm}, 12.0 \mathrm{~nm}$, and $12.9 \mathrm{~nm}(0.286 \mathrm{~nm}$ rise/residue for collagen triple helices). ${ }^{[8]}$ The heights obtained from AFM confirm that the nanosheets comprise a single layer with triple helices packed perpendicular to the surface. Altogether, these results demonstrate that one can systematically tune CMP nanosheets in all dimensions simply through extending the length of the triple helical building block.

Circular dichroism (CD) spectropolarimetry of CMP nanosheets displays the characteristic collagen triple helix profile marked by a positive maximum at $\sim 224 \mathrm{~nm}$ and a negative minimum at $\sim 198 \mathrm{~nm}$ (Figure 3). Rpn values, which report the ratio of these peak intensities, are useful in determining the formation of collagen triple helices in solution. ${ }^{[9]}$ The values confirm that the triple helices are present for all optimized CMP assemblies (Rpn $\geq 0.09)$ except for $\mathbf{4 S}(\mathbf{X})_{\mathbf{4 3 4}}(4$ $\mathrm{mg} / \mathrm{mL}, \mathrm{Rpn}=0.05){ }^{[9]}$

It is well-known that increasing the Pro-Hyp-Gly length within collagen peptides increases the stability of the triple helix. ${ }^{[10]}$ Thermal denaturation studies of $\mathbf{4 S}(X)_{444}, \mathbf{4 S}(X)_{454}, \mathbf{4 S}(X)_{464}$, and $4 \mathrm{~S}(\mathrm{X})_{474}$ sheets reveal melting temperatures $\left(\mathrm{T}_{\mathrm{m}}\right)$ of $34^{\circ} \mathrm{C}, 50^{\circ} \mathrm{C}$, $44^{\circ} \mathrm{C}$, and $49^{\circ} \mathrm{C}$, respectively (Figure S8). The lower $T_{m}$ of $\mathbf{4 S}(X)_{\mathbf{4 6 4}}$ and $\mathbf{4 S}(X)_{474}$, compared to $\mathbf{4 S}(X)_{454}$, is attributed to the lower [CMP]. In order to allow for direct comparison of the triple helix stability between CMPs, $T_{m}$ values were acquired on preassembled CMP nanosheets at a single concentration (1 $\mathrm{mg} / \mathrm{mL}$; Figure S9). Thermal transitions at $22^{\circ} \mathrm{C}$ and $36^{\circ} \mathrm{C}$ for $\mathbf{4 S}(X)_{444}$ and $\mathbf{4 S}(X)_{454}$, respectively, provide further evidence that shorter CMPs, having fewer Pro-Hyp-Gly triads, are less stable than their longer CMP counterparts.

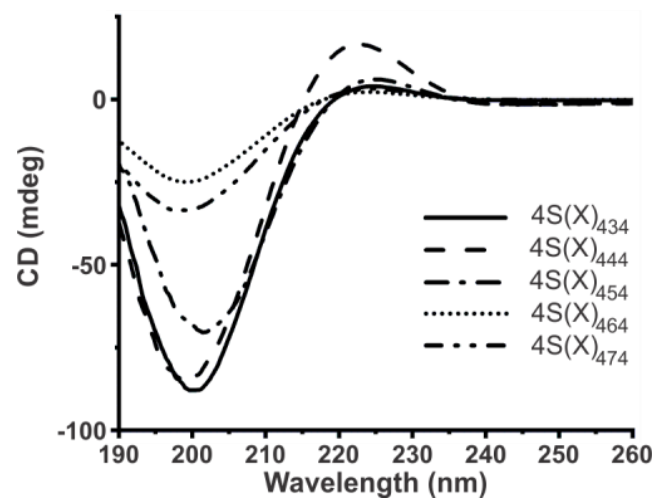

Figure 3. CD spectra of assembly solutions of $\mathbf{4 S}(\mathbf{X})_{434}, 4 \mathrm{mg} / \mathrm{mL}, R p n=0.05$ $\mathbf{4 S}(\mathbf{X})_{444}, 4 \mathrm{mg} / \mathrm{mL}$, Rpn $=0.19 ; \mathbf{4 S}(\mathbf{X})_{454}, 4 \mathrm{mg} / \mathrm{mL}, \mathrm{Rpn}=0.09 ; \mathbf{4 S}(\mathbf{X})_{464}, 1$ $\mathrm{mg} / \mathrm{mL}, \mathrm{Rpn}=0.09 ;$ and $\mathbf{4 S}(\mathbf{X})_{474}, 1 \mathrm{mg} / \mathrm{mL}, \mathrm{Rpn}=0.10$.

We hypothesized that the change in size over the $\mathbf{4 S}(\mathbf{X})$ series is likely due to the frequency of nucleation events that occur during the assembly process. Moreover, TEM data reveal differences in sheet homogeneity between CMP assemblies. To address these discrepancies, timepoint studies were conducted (Figure $4 \mathrm{a}$ and $\mathbf{S 1 0 - 1 2 ) . ~ S m a l l ~} \mathbf{4 S}(\mathbf{X})_{444}$ nanosheets are observed as early as $30 \mathrm{~min}$ after reaching the incubation temperature of $4^{\circ} \mathrm{C}$. Subsequent timepoints reveal that the sheets appear to grow uniformly and reach maturity after 2 weeks (Figure 4a). Similarly, $\mathbf{4 S}(\mathbf{X})_{454}, \mathbf{4 S}(X)_{464}$, and $\mathbf{4 S}(\mathbf{X})_{474}$ nanosheets are observed at early timepoints at room temperature and grow over time; however, the sheets appear to be less homogenous throughout the assembly process (Figures S10-12).

To quantify these results, we plotted nanosheet size as a function of time along with the corresponding relative standard deviation (RSD; Figures $4 \mathrm{~b}$ and S13). RSD values were calculated to determine the degree of dispersity of the sheet population. Sheets grew in a logarithmic fashion regardless of CMP length. Starting RSD value of $37 \%$ was observed for $\mathbf{4 S}(\mathbf{X})_{444}$, followed by an exponential decrease in a manner that is inversely proportional to growth in sheet size (Figure $4 b$ ). The decrease in RSD values implies that no sheet nucleation occurs after the initial nucleation event. As the sheets grow the difference in sheet sizes become less significant leading to smaller RSD values.
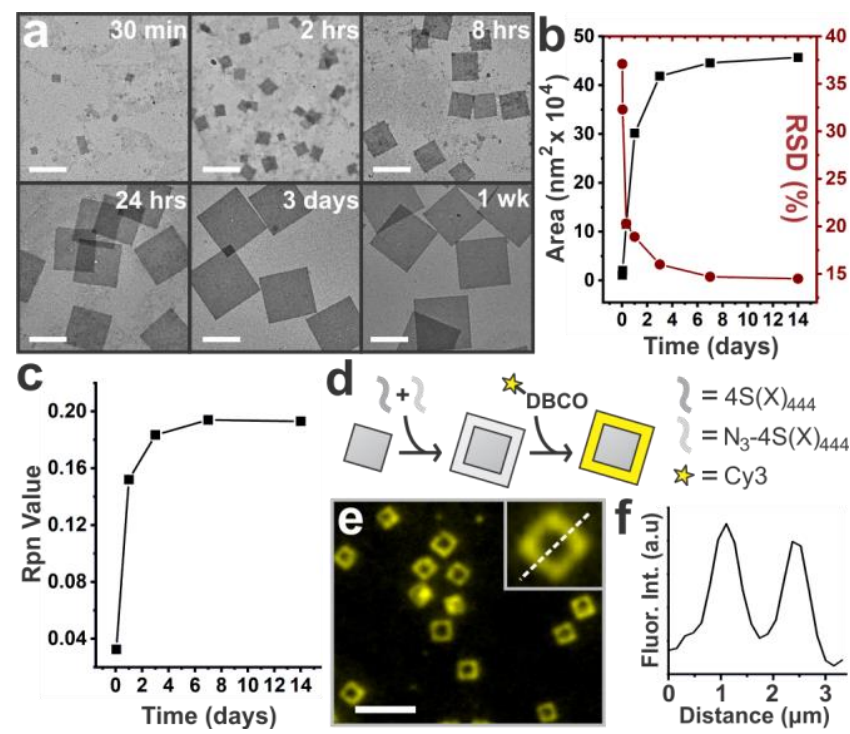

Figure 4. (a) TEM images of $\mathbf{4 S}(X)_{\mathbf{4 4 4}}$ nanosheets at various timepoints over the first week of assembly at $4^{\circ} \mathrm{C}$ (scale bar $=500 \mathrm{~nm}$ ). (b) Square area of $\mathbf{4 S}(X)_{444}$ nanosheets and corresponding RSD values as a function of assembly time. (c) Rpn values of $\mathbf{4 S}(X)_{444}$ assembly solution as a function of assembly time. (d) Homoepitaxial growth of $\mathbf{4 S}(X)_{444}$ and $\mathbf{N}_{\mathbf{3}}-\mathbf{4 S}(X)_{\mathbf{4 4 4}}$ monomers (9:1) around preformed $\mathbf{4 S}(X)_{\mathbf{4 4 4}}$ sheets followed by attachment of Cy3 via copperfree azide-alkyne cycloaddition. (e) Fluorescence optical micrograph after 1 week of growth reveals the successful homoepitaxy off $\mathbf{4 S}(X)_{\mathbf{4 4 4}}$ nanosheet seeds (scale bar $=5 \mu \mathrm{m}$ ). False color is used to enhance contrast. (f) Fluorescence line scan of the nanosheet shown in the inset of $(e)$.

In contrast, RSD values of $\mathbf{4 S}(X)_{454}, \mathbf{4 S}(X)_{464}$, and $\mathbf{4 S}(X)_{474}$ nanosheets over the 2-week time study remain relatively constant, suggesting that nanosheet seeds continue forming throughout the assembly process (Figure S13). Analysis of distribution data of sheet sizes collected at each timepoint confirms the assembly discrepancies between CMPs (Figure S14). While monodisperse nanosheet seeds are formed within 2 hrs for all CMPs, frequent nucleation events for $\mathbf{4 S}(X)_{464}$ and $\mathbf{4 S}(\mathbf{X})_{474}$ assemblies continue to take place throughout the assembly process. $\mathbf{4 S}(\mathbf{X})_{444}$ seeds, however, are almost entirely formed only during the initial nucleation event (within $2 \mathrm{hrs}$ ). The 
initial nanosheets generated at these early timepoints serve as seeds for homoepitaxial addition of collagen triple helices. $\mathbf{4 S}(\mathbf{X})_{\mathbf{4 5 4}}$ follows a similar assembly process as $\mathbf{4 S}(\mathbf{X})_{\mathbf{4 4 4}}$, however, sheet nucleation continues to occur after the initial timepoints, though less frequently than observed for the longer CMPs.

In addition to TEM, CD timepoint studies were also conducted to monitor the formation of collagen triple helices in solution (Figure S15). Interestingly, Rpn values, which provide feedback on triple helix formation, increase over time in a manner comparable to sheet growth, implying that sheet growth is controlled by triple helix formation or vice versa (Figures $4 \mathrm{c}$ and $\mathrm{S} 16)$. This codependence may be due to the stabilization of triple helix folding offered by the $2 \mathrm{D}$ lattice of the nanosheet assembly, which counteracts the unfavorable charge repulsion between parallel strands within isolated CMP triple helices.

From these time-dependent studies, we conclude that the homogeneity and lateral dimensions of nanosheets are nucleation-dependent, and that tuning the length of the central block regulates the generation of sheet nuclei, which controls sheet size and dispersity. We hypothesize that $\mathbf{4 S}(X)_{444}$ happens to be at the "sweet spot", i.e., the position in which nucleation of the sheets and subsequent growth off those sheets is controlled. This fortuitous situation gives rise to unusually monodisperse nanosheets. The assembly process resembles crystallizationdriven living polymerization, where seed edges remain active to epitaxial growth of successive additions of monomers. ${ }^{[3 e, 11]}$ To probe whether $\mathbf{4 S}(\mathbf{X})_{444}$ nanosheets are 'living,' a 9:1 mixture of $\mathbf{4 S}(X)_{444}$ and $\mathrm{N}$-terminal azido-modified $\mathbf{4 S}(X)_{444}$ (azido-PEG5acid, $\mathbf{N}_{3}-\mathbf{4 S}(\mathbf{X})$; Figure $\left.\mathrm{S} 17\right)$ monomers were introduced to a solution of pre-assembled $\mathbf{4 S}(\mathbf{X})_{\mathbf{4 4 4}}$ nanosheets. The azide derivative allows for the postsynthetic conjugation of Су3 fluorophore onto the sheet surface via copper-free azide-alkyne cycloaddition (Figure 4d). TEM and Fluorescence microscopy of nanosheets after 1 week reveals the successful homoepitaxy (Figures 4e,f and S18,19), unveiling an additional route for fabricating size-tunable CMP nanosheets as well as highlighting the potential for functionalizing the sheet surface with nanoscale spatial control. In summary, we suggest that $\mathbf{4 S}(X)_{444}$ has a diminished ability to generate new nuclei compared to the more crystallizable triple helices of longer CMPs. This allows for a controlled growth process in which newly formed triple helices attach onto existing nanosheets leading to a more uniform product.

Small- and Wide-angle X-ray scattering (SAXS/WAXS) experiments were conducted on the nanosheets to characterize their internal structure. We hypothesized that differences in lattice packing parameters and degree of crystallinity could contribute to the observed variation in sheet size. Scattering curves were obtained for all CMP nanosheets, including $\mathbf{4 S}(X)_{434}$ nanosheets $(8 \mathrm{mg} / \mathrm{mL}$; Figure $5 \mathrm{a})$. The scattering intensities at low $q$ region $\left(q<0.02 \AA^{-1}\right)$ roughly follow $q^{-2}$ power law, indicating a uniform sheet-like morphology for all CMP nanosheets except those derived from $\mathbf{4 S}(\mathbf{X})_{\mathbf{4 3 4}}$, which is likely due to multilamellar stacking. Fitting the low $q$ data using the Guinier equation for sheet-like forms, average sheet thicknesses are obtained that are consistent with AFM and theoretical height calculations (105.9 A, $119.3 \AA$, $128.5 \AA$, $134.7 \AA$; Figure S20). The oscillations in the $q$ region of $\sim 0.03-0.3 \AA^{-1}$ arise from the thickness of the nanosheets and the attenuation of the oscillation reflects the variation of nanosheet thickness, which is estimated as ca. $15 \AA$ (Figure S21). ${ }^{[4]}$

All CMP nanosheets possess a high degree of crystallinity as observed from the presence of several Bragg diffraction peaks in the high $q$ region (i.e. small $d$-spacing) of the intensity plot (Figure 5a). Unsurprisingly, the peak patterns are comparable indicating that they exhibit a similar underlying assembly structure. Peaks (1) and (2), observed for all CMP sheets, correspond to $d$-spacings that range from $14.1 \AA$ to $13.3 \AA$ and $10.0 \AA$ to $9.7 \AA$, respectively. These sets of peaks are related in that peak (2) multiplied by $\sqrt{ } 2$ is roughly equal to peak (1), which suggests the presence of ordered tetragonal packing of collagen triple helices within the assemblies. This relation holds well for $\mathbf{4 S}(X)_{444}$ and $\mathbf{4 S}(X)_{454}$ nanosheets, but to a lesser extent for nanosheets derived from $\mathbf{4 S}(X)_{464}$ and $\mathbf{4 S}(X)_{474}$, implying that the tetragonal lattices of the latter are slightly distorted. Furthermore, the results reveal that the lattice contracts with each Pro-HypGly addition to the core block (Figure $5 b$ ). We note that the WAXS data for $\mathbf{4 S}(\mathbf{X})_{\mathbf{4 4 4}}$ is slightly different than that previously reported. ${ }^{[4]}$ We ascribe these differences to the previously reported sheets not having reached full maturity.
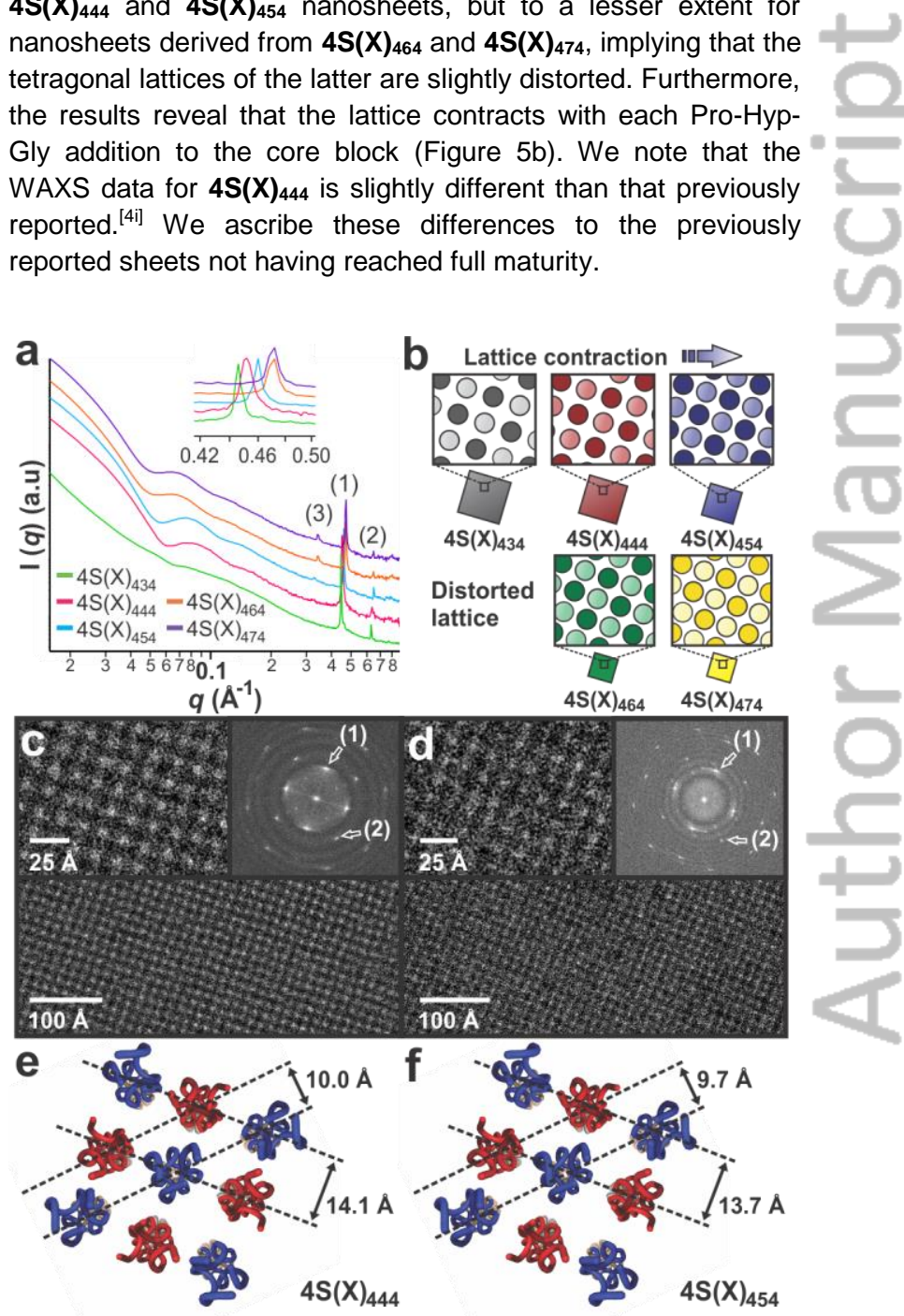

Figure 5. (a) Synchrotron SAXS scattering profile. Peak (1) $d$-spacings: $\mathbf{4 S}(\mathbf{X})_{\mathbf{4 3 4}}, 14.1 \AA ; \mathbf{4 S}(\mathbf{X})_{444}, 13.9 \AA ; \mathbf{4 S}(X)_{454}, 13.7 \AA ; \mathbf{S S}(X)_{464}, 13.4 \AA ; \mathbf{S S}(X)_{474}$, 13.3 $\AA$. Peak (2) d-spacings: $4 \mathbf{S}(X)_{434}, 10.0 \AA$; $\mathbf{4 S}(X)_{444}, 9.9 \AA$; $\mathbf{4 S}(X)_{454}, 9.7 \AA$; $\mathbf{4 S}(\mathbf{X})_{464}, 9.7 \AA ; \mathbf{S}(\mathbf{X})_{474}, 9.7 \AA$. . Peak (3) d-spacings: $\mathbf{4 S}(\mathbf{X})_{\mathbf{4 5 4}}, 19.2 \AA$ $\mathbf{4 S}(\mathbf{X})_{464}, 18.5 \AA ; \mathbf{4 S}(\mathbf{X})_{474}, 18.5 \AA$. Inset: expansion of peak (1) diffraction peaks. (b) Cartoon representations of the different lattice packing parameters between CMP nanosheets. (c) High-resolution cryo-TEM images and corresponding FFT analysis of (c) $\mathbf{4 S}(X)_{444}$ and (d) $\mathbf{4 S}(X)_{454}$ nanosheets. FFT spots (d-spacing) for $\mathbf{4 S}(\mathbf{X})_{444}$ : (1) $14.1 \AA$, $10.0 \AA$ and $\mathbf{4 S}(\mathbf{X})_{454}:$ (1) $13.7 \AA$, (2) 
9.7 A. Assembly model of the 2D lattice of $(e) \quad \mathbf{S S}(X)_{444}$ and (f) $4 \mathbf{S}(X)_{454}$ nanosheets.

The SAXS/WAXS data suggest that the longer CMP sequences allow the protomers to pack more tightly to one another within the 2D lattice. Close examination of peak (3), found at smaller $q$ values, reveals that this peak becomes more prominent with increasing CMP length - a sign that the nanosheet exhibits greater internal crystallinity at longer lengthscales. Moreover, the lattice distortion observed for $\mathbf{4 S}(X)_{464}$ and $\mathbf{4 S}(X)_{474}$ is reminiscent of single-crystal X-ray diffraction studies of synthetic collagen triple helices, which often pack into layered structures displaying distorted pseudo-tetragonal or hexagonal packing symmetry. ${ }^{[12]}$ We hypothesize that as the central block becomes longer, its structure increasingly dominates the 2D packing behavior of the triple helices, which deviates from a purely tetragonal lattice. Altogether, these results correspond well with the previous results in that longer CMPs generate sheet nuclei more readily than their shorter CMP counterparts.

Cryogenic transmission electron microscopy (cryo-TEM) with direct electron detection was employed on $\mathbf{4 S}(X)_{444}$ and $\mathbf{4 S}(X)_{454}$ nanosheets to study in further detail their internal structure (Figures $5 \mathrm{c}, \mathrm{d}$ and S22,23). These peptides were chosen as substrates because the larger size of the corresponding nanosheets facilitates visualization under the microscope. Highresolution cryo-TEM images reveal strikingly ordered tetragonal packing of individual triple helices within the nanosheet assembly, in which individual distances between triple helical protomers can be determined directly in real-space.

Fast Fourier transformations (FFT) of the cryo-TEM images confirm the high degree of order within the nanosheets with Bragg spots observed up to $d$-spacings of $\sim 4.5 \AA$ (Figures $\mathrm{S} 22,23)$. The presence of Thon rings at resolutions outside the computed Bragg spots indicates the breakdown of 2D order beyond that resolution. A 4-fold rotational symmetry confirms the tetragonal lattice of both CMP assemblies. Measured $d$-spacings of $\sim 14.1 \AA$ and $\sim 10.0 \AA$ for $\mathbf{4 S}(\mathbf{X})_{444}$ nanosheets, and $\sim 13.7 \AA$ and $\sim 9.7 \AA$ for $\mathbf{4 S}(\mathbf{X})_{454}$ nanosheets are in close agreement with corresponding $d$-spacings obtained from the 1D SAXS curves. To account for any distortions of Bragg spots arising from their possible location on Thon rings, images obtained with similar defocus distances were used to allow for direct comparison.

Based on the structural information obtained from AFM, SAXS and cryo-TEM, we propose assembly models for $\mathbf{4 S}(X)_{444}$ and $\mathbf{4 S}(\mathbf{X})_{454}$ nanosheets (Figure $\left.5 \mathrm{e}, \mathrm{f}\right)$. The nanosheets comprise a single 2D tetragonal lattice of collagen triple helices that are oriented perpendicular to the sheet surface. The helices pack in an antiparallel fashion allowing oppositely charged triads from neighboring triple helices to be in contact, which provides the cohesive electrostatic interactions that stabilize the triple helices and the corresponding $2 \mathrm{D}$ assemblies. We note that the model for $\mathbf{4 S}(\mathbf{X})_{444}$ sheets corrects a previously proposed model, ${ }^{[4]}$ highlighting the importance of real-space imaging obtainable by cryo-TEM compared to SAXS measurements in which the data are azimuthally averaged. Furthermore, we can propose a model for $\mathbf{4 S}(\mathbf{X})_{\mathbf{4 3 4}}$ nanosheets, which is simply an expansion of the $\mathbf{4 S}(X)_{444}$ lattice. While at present, assembly models for $\mathbf{4 S}(X)_{464}$ and $\mathbf{4 S}(X)_{474}$ assemblies cannot be constructed with full certainty, the insights obtained from this study allows us to propose that their lattices are nearly identical and are slightly distorted and contracted compared to the rest of the $\mathbf{4 S}(\mathrm{X})$ family. These structural distortions may reflect the intrinsic packing preference of the longer Pro-Hyp-Gly block within the triblock sequence architecture (vide supra).

In conclusion, we demonstrate hierarchical structural control of nanosheets through systematic changes within the triple helix architecture. We show that sequence-directed self-assembly of cylindrical rod-like units, such as collagen triple helices, represents a facile mechanism for the construction of structurally defined and tunable 2D assemblies. The results suggest that supramolecular interactions between structural elements can be propagated hierarchically to afford structural control across length-scales. Furthermore, we demonstrate homoepitaxial growth of $\mathbf{4 S}(\mathbf{X})_{\mathbf{4 4 4}}$ nanosheets, which represents an intriguing and potentially useful method for incorporating multiple functional domains within the assembly architecture with nanoscale resolution. Lastly, we anticipate these results will afford general assembly guidelines for engineering 2D systems that are built from rigid, helical structural motifs and offer instruction for controlling their assembly parameters across a range of length-scales.

\section{Acknowledgements}

This work was supported by the NSF (CHE-1808509, V.P.C), and used resources of the Advanced Photon Source, a U.S. DOE Office of Science User Facility operated for the DOE Office of Science by Argonne National Laboratory under contract No. DE-AC02-06CH11357. We thank Prof. Tianquan Lian and Prof. Brian Dyer for use of the atomic force microscope and DLS, respectively. We also acknowledge the following people: Lubomir Kovacik and Henning Stahlberg for their help with microscope operation and use of the cryo-TEM facility, respectively.

Keywords: Collagen-mimetic peptides $\cdot$ nanoarchitectonics $•$ nanosheets $\cdot$ self-assembly $\bullet$ biomaterials

[1] a) H. Zhang, ACS Nano 2015, 9, 9451-9469; b) X. Zhuang, Y. Mai, D. Wu, F. Zhang, X. Feng, Adv. Mater. 2015, 27, 403-427; c) T. Govindaraju, M. B. Avinash, Nanoscale 2012, 4, 6102-6117; d) C. E. Boott, A. Nazemi, I. Manners, Angew. Chem. Int. Ed. 2015, 54, 1387613894; e) J. W. Colson, W. R. Dichtel, Nat. Chem. 2013, 5, 453; f) S. Zhang, J. Zhang, W. Fang, Y. Zhang, Q. Wang, J. Jin, Nano Lett. 2018, 18,6563-6569; g) C.-A. Palma, P. Samorì, Nat. Chem. 2011, 3, 431; h) X. Zhang, Y. Xie, Chem. Soc. Rev. 2013, 42, 8187-8199; i) J. Sakamoto, J. van Heijst, O. Lukin, A. D. Schlüter, Angew. Chem. Int. Ed. 2009, 48, 1030-1069.

[2] K. S. Novoselov, A. K. Geim, S. V. Morozov, D. Jiang, Y. Zhang, S. V. Dubonos, I. V. Grigorieva, A. A. Firsov, Science 2004, 306, 666-669.

[3] a) L. Han, M. Wang, X. Jia, W. Chen, H. Qian, F. He, Nat. Commun. 2018, 9, 865; b) X. He, M.-S. Hsiao, Charlotte E. Boott, Robert L. Harniman, A. Nazemi, X. Li, Mitchell A. Winnik, I. Manners, Nat. Mater. 2017, 16, 481; c) A. Nazemi, X. He, L. R. MacFarlane, R. L. Harniman, M.-S. Hsiao, M. A. Winnik, C. F. J. Faul, I. Manners, J. Am. Chem. Soc. 2017, 139, 4409-4417; d) H. Qiu, Y. Gao, C. E. Boott, O. E. C. Gould, R. L. Harniman, M. J. Miles, S. E. D. Webb, M. A. Winnik, I. Manners, 
Science 2016, 352, 697; e) B. Yu, X. Jiang, J. Yin, Macromolecules 2014, 47, 4761-4768; f) M. Inam, G. Cambridge, A. Pitto-Barry, Z. P. L. Laker, N. R. Wilson, R. T. Mathers, A. P. Dove, R. K. O'Reilly, Chem. Sci. 2017, 8, 4223-4230.

[4] a) H.-S. Jang, J.-H. Lee, Y.-S. Park, Y.-O. Kim, J. Park, T.-Y. Yang, K. Jin, J. Lee, S. Park, J. M. You, K.-W. Jeong, A. Shin, I.-S. Oh, M.-K. Kwon, Y.-I. Kim, H.-H. Cho, H. N. Han, Y. Kim, Y. H. Chang, S. R. Paik, K. T. Nam, Y.-S. Lee, Nat. Commun. 2014, 5, 3665; b) J. Lee, I. R. Choe, N.-K. Kim, W.-J. Kim, H.-S. Jang, Y.-S. Lee, K. T. Nam, ACS Nano 2016, 10, 8263-8270; c) Y. Lin, M. R. Thomas, A. Gelmi, V. Leonardo, E. T. Pashuck, S. A. Maynard, Y. Wang, M. M. Stevens, J. Am. Chem. Soc. 2017, 139, 13592-13595; d) B. Dai, D. Li, W. Xi, F Luo, X. Zhang, M. Zou, M. Cao, J. Hu, W. Wang, G. Wei, Y. Zhang, C. Liu, Proc. Natl. Acad. Sci. U.S.A 2015, 112, 2996-3001; e) A. S. Parmar J. K. James, D. R. Grisham, D. H. Pike, V. Nanda, J. Am. Chem. Soc 2016, 138, 4362-4367; f) I. W. Hamley, A. Dehsorkhi, V. Castelletto, Chem. Commun. 2013, 49, 1850-1852; g) H. V. Zhang, F. Polzer, M. J. Haider, Y. Tian, J. A. Villegas, K. L. Kiick, D. J. Pochan, J. G. Saven, Sci. Adv. 2016, 2, e1600307; h) T. Jiang, C. Xu, Y. Liu, Z. Liu, J. S. Wall, X. Zuo, T. Lian, K. Salaita, C. Ni, D. Pochan, V. P. Conticello, J. Am. Chem. Soc. 2014, 136, 4300-4308; i) T. Jiang, C. Xu, X. Zuo, V. P. Conticello, Angew. Chem. Int. Ed. 2014, 53, 8367-8371; j) E. L. Magnotti, S. A. Hughes, R. S. Dillard, S. Wang, L. Hough, A Karumbamkandathil, T. Lian, J. S. Wall, X. Zuo, E. R. Wright, V. P. Conticello, J. Am. Chem. Soc. 2016, 138, 16274-16282.

[5] a) E. J. Robertson, A. Battigelli, C. Proulx, R. V. Mannige, T. K. Haxton, L. Yun, S. Whitelam, R. N. Zuckermann, Acc. Chem. Res. 2016, 49 379-389; b) E. J. Robertson, G. K. Olivier, M. Qian, C. Proulx, R. N. Zuckermann, G. L. Richmond, Proc. Natl. Acad. Sci. 2014, 111, 13284
13289; c) H. Jin, F. Jiao, M. D. Daily, Y. Chen, F. Yan, Y.-H. Ding, X. Zhang, E. J. Robertson, M. D. Baer, C.-L. Chen, Nat. Commun. 2016, 7, 12252; d) K. T. Nam, S. A. Shelby, P. H. Choi, A. B. Marciel, R. Chen, L. Tan, T. K. Chu, R. A. Mesch, B.-C. Lee, M. D. Connolly, C. Kisielowski, R. N. Zuckermann, Nat. Mater. 2010, 9, 454.

[6] a) Y. He, Y. Chen, H. Liu, A. E. Ribbe, C. Mao, J. Am. Chem. Soc. 2005, 127, 12202-12203; b) W. Liu, H. Zhong, R. Wang, N. C. Seeman, Angew. Chem. Int. Ed. 2011, 50, 264-267; c) P. Wang, S. Gaitanaros, S. Lee, M. Bathe, W. M. Shih, Y. Ke, J. Am. Chem. Soc. 2016, 138, 7733-7740; d) H. Yu, D. T. L. Alexander, U. Aschauer, R. Häner, Angew. Chem. Int. Ed. 2017, 56, 5040-5044.

[7] A. Lampel, R. V. Ulijn, T. Tuttle, Chem. Soc. Rev. 2018, 47, 3737-3758.

[8] a) K. Okuyama, Connect. Tissue Res. 2008, 49, 299-310; b) K. Okuyama, X. Xu, M. Iguchi, K. Noguchi, Peptide Sci. 2006, 84, 181-191.

[9] Y. Feng, G. Melacini, J. P. Taulane, M. Goodman, J. Am. Chem. Soc. 1996, 118, 10351-10358.

[10] A. V. Persikov, J. A. M. Ramshaw, B. Brodsky, J. Biol. Chem. 2005, 280, 19343-19349.

[11] a) Z. M. Hudson, C. E. Boott, M. E. Robinson, P. A. Rupar, M. A. Winnik, I. Manners, Nat. Chem. 2014, 6, 893; b) J. B. Gilroy, T. Gädt, G. R. Whittell, L. Chabanne, J. M. Mitchels, R. M. Richardson, M. A. Winnik, I. Manners, Nat. Chem. 2010, 2, 566.

[12] a) K. Okuyama, C. Hongo, G. Wu, K. Mizuno, K. Noguchi, S. Ebisuzaki, Y. Tanaka, N. Nishino, H. P. Bächinger, Biopolymers 2009, 91, 361 372; b) K. Okuyama, K. Miyama, K. Mizuno, H. P. Bächinger, Biopolymers 2012, 97, 607-616; c) C. Hongo, K. Noguchi, K. Okuyama Y. Tanaka, N. Nishino, J. Biochem. 2005, 138, 135-144; d) R. Berisio, L. Vitagliano, L. Mazzarella, A. Zagari, Protein Sci. 2002, 11, 262-270. 
Entry for the Table of Contents (Please choose one layout)

Layout 1:

\section{COMMUNICATION}

Nanosheet Engineering: Fine-tuning of $2 \mathrm{D}$ crystalline nanosheets across the sub-nano- and mesoscale regime is achieved via systematically adjusting the length and stability of collagen triple helices. The results afford broad engineering principles for the design and construction of tailorable 2D nanomaterials assembled from rigid, helical structural motifs (e.g. a-helices, coiled coils, etc.).

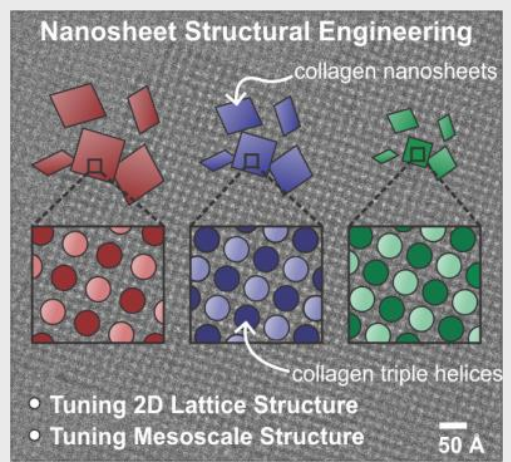

Andrea D. Merg, Gavin Touponse, Eric van Genderen, Xiaobing Zuo, Alisina Bazrafshan, Thorsten Blum, Spencer Hughes, Khalid Salaita, Jan Pieter Abrahams, Vincent $P$. Conticello*

Page No. - Page No.

2D Crystal Engineering of Nanosheets Assembled from Helical Peptide Building Blocks 